\title{
Evolution of Two-Dimensional Electromagnetic Devices Using a Novel Genome Structure
}

\author{
Mark Dorica and Dennis D. Giannacopoulos \\ Department of Electrical \& Computer Engineering, McGill University, Montreal, Quebec H3A 2A7, Canada
}

\begin{abstract}
The majority of evolutionary algorithm applications in electromagnetic design have focused on optimization of device dimensions. This approach preconceives the final device shape. To facilitate the evolution of complex and creative designs through genetic algorithms, a novel genome and genetic operators are introduced. They are then used to evolve a patch antenna for dual-frequency operation. The final design that emerges has a fitness roughly triple that of the standard approach.
\end{abstract}

Index Terms-Electromagnetic optimization, genetic algorithms (GAs), patch antennas.

\section{INTRODUCTION}

G ENERATING designs through genetic algorithms (GAs) has had much success in recent years [1]. The vast majority of design problems that are tackled with GAs place a heavy emphasis on the optimization of preconceived shapes through parametrization of device dimensions. In this direct encoding scheme, each feature in the phenotype (device) is directly represented in the genotype.

There are alternate ways of performing the mapping between the genome on which the GA operates and the final design. Much of this work involving artificial embryogenies (AE) is focused on achieving high levels of complexity (e.g., large commercial buildings [2]), while avoiding an intractable genotypic search space. Many of the recent contributions either deal with cell-chemistry approaches, which mimic how physical structures emerge in biology, or grammatical approaches, which evolve sets of rules in the form of grammatical rewrite systems [2]. A major difficulty is in the design of these AEs, which continues to be an art.

Consider the design of 2-D metal structures that can be fully described in a grid. Placing atoms in this 2-D design space can lead to all possible designs of structures. The obvious direct mapping between genes and atoms would of course lead to an intractable genotypic search space. This fully general approach is not within our grasp anyway due to limitations in manufacturing technology. But if huge advances in complexity can be achieved by transforming the mapping between genotype and phenotype [2], then perhaps other advantages can also be obtained through the use of novel AEs.

\section{TRaditional Parametric Design}

The E-shaped patch antenna, shown in Fig. 1, is a well-known configuration [3]. It has an interesting feature in its two slots. These slots have the effect of increasing the path of the current

Digital Object Identifier 10.1109/TMAG.2006.892106

Color versions of one or more of the figures in this paper are available online at http://ieeexplore.org.
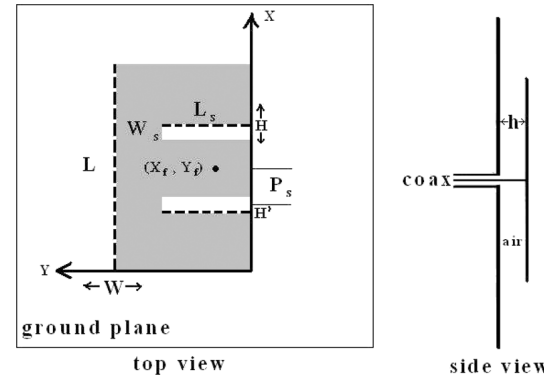

Fig. 1. Geometry of E-shaped patch antenna.

from the feeding point to the top and bottom sections of the antenna, causing a dual resonant behavior [3].

Earlier work on parametric optimization of this configuration $\left(\mathrm{H}, \mathrm{H}^{\prime}\right.$, and $\mathrm{W}$ parameters in Fig. 1) yielded dual-frequency operation at 2.21/2.58 GHz [4]. Adapting the design to new requirements is not necessarily straightforward; using the same starting design and ranges as in [4], would lead to unsatisfactory optimization results for other frequencies, such as $3.5 / 4.5 \mathrm{GHz}$. One of the main obstacles with this parametric approach is the expert knowledge required for selecting the initial configuration for a particular design problem. To overcome this obstacle, more versatile mappings are desirable [5]-[7]. In subsequent sections we will investigate whether novel genome structures are able to overcome the limitations associated with the parametric optimization of this antenna shape.

\section{BINARY GRID DESIGN}

The binary grid approach is a step toward removing constraints on the final shape of the device. The concept is straightforward; to divide the design space into a grid of cells. The subsequent GA optimization operates on a binary genome where each bit represents one of the cells. This "on/off" approach to placement of metal has been applied successfully in the design of a multitude of devices, including patch antennas [5], [6], and filters [7].

\section{NEW QuAdTREe-BASEd GENOME}

One major drawback of the binary grid approach is that the level of discretization must be pre-defined. A very fine 

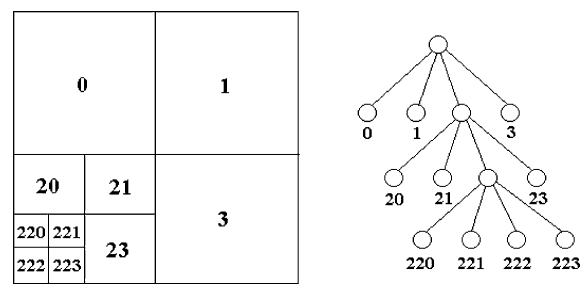

Fig. 2. Quadtree data structure.

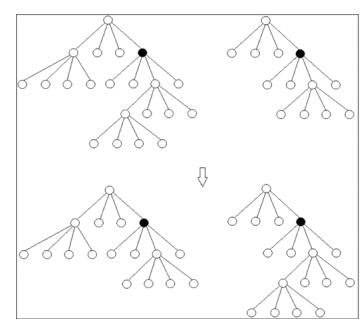

(a)

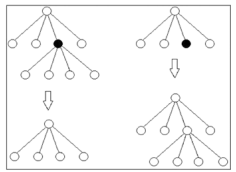

(b)
Fig. 3. Crossover operator (a) and structural mutation operators (b), adapted for quadtree-based genome.

discretization may lead to an extensive search time, and a very coarse discretization may not be able to reach the level of detail required to meet specifications. There may be performance benefits in evolving not only structural details, but also the representation of the structure within the genome. Hence, we introduce a quadtree-based genome (Fig. 2).

The quadtree is based on a recursive decomposition of space. It yields a repetitive pattern, where increased resolution implies increasing the depth of the tree. Through variations in its tree structure, this form of variable-length genome allows search through new and different search spaces. This is in contrast to the fixed binary genome search space. To enable quadtree evolution, specialized crossover and mutation operators were implemented.

A standard tree-based crossover operator would select two nodes at random (one in each parent), and create offspring by swapping subtrees. This approach would not be appropriate here because features from different sections of the design space would be exchanged. The solution is to assign to each node a code as shown in Fig. 2. A crossover site can then be selected in one parent, and crossover only proceeds if the same code is found in the second parent.

Three mutation operators, designed to operate on subtrees and on terminal nodes, were implemented. The structural "additive" and "destructive" mutators are shown in Fig. 3(b). These mutators have the ability to alter the levels of discretization of large sections of the design. The additive mutator replaces a terminal node by a random single level subtree. The destructive mutator is analogous except that the subtree is deleted and the remaining node is assigned a random bit. A third "terminal flip" mutator flips the binary values of the terminal nodes according to the mutation probability. For a given call of the mutation routine, only a single one of these mutators is executed.

\section{Performance of New Genome}

The proposed system is intended to allow the discovery of an efficient problem specific genotype-device mapping while not excluding any potential solutions. The GA is steady-state with
TABLE I

Designs From GenOME STRUCTURES

\begin{tabular}{lccc}
\hline Genome & Mean fitness & Mean simulations & Mean time cost \\
\hline Quadtree & $17.93 \mathrm{~dB}$ & 1003 & $98.9 \mathrm{mins}$ \\
\hline Binary & $6.52 \mathrm{~dB}$ & 1100 & $95.2 \mathrm{mins}$ \\
\hline
\end{tabular}

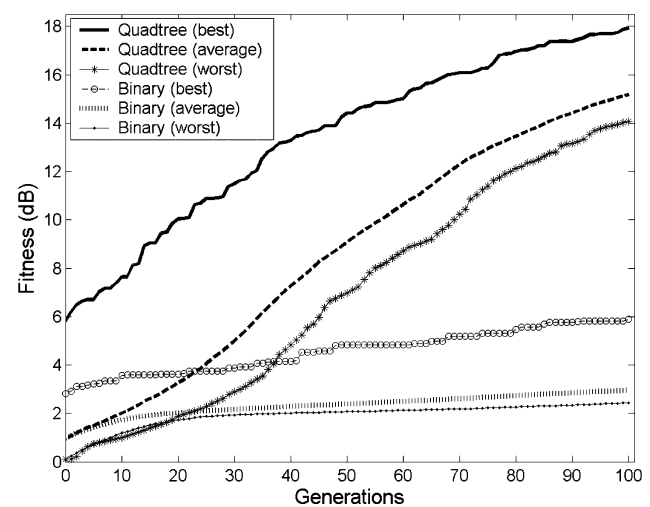

Fig. 4. Evolutionary performance of genome structures.

replacement set to 0.1 . The population and number of generations are both 100. Crossover and mutation probabilities are 0.7 and 0.05 , respectively. Note that with the variety of mutation operators, the mutation probability must be interpreted accordingly. The GA operates on a tree of maximum depth 5 (including the root node), which implies a maximum discretization of 16 $\times 16$ cells in the design space.

Tests were carried out for a $32 \times 32 \mathrm{~mm}$ design space. For symmetry, the second half of the antenna is obtained by reflection. The antenna is fed at the center of the full region. The goal of the design process is to optimize the worst $\left|\mathrm{S}_{11}\right|(\mathrm{dB})$ at 2 frequencies: $3.5 \mathrm{GHz}$ and $4.5 \mathrm{GHz}$. Symbolically, the objective function to be minimized is

$$
\text { objective }=\max _{3.5,4.5 \mathrm{GHz}}\left(\left|S_{11}\right|_{d B}\right) .
$$

Table I shows an almost threefold advantage of the hierarchical quadtree approach over the standard binary encoding, for similar simulation time costs. The term "fitness" is simply the absolute value of the objective function (1). Note that the number of simulations is slightly higher for the binary approach. With 256 bits and a mutation probability set to 0.05 , a bit flip is almost always executed in the binary genome, triggering a re-evaluation of the fitness. Because of its variable length, the quadtree genome is less likely to be modified through mutation. Still, the time costs are comparable partly because of the extra overhead associated with the specialized crossover and mutation operators of the quadtree genome. This is an intrinsic cost of advanced mappings.

Other results that compare the binary encoding with the new quadtree approach are shown in Fig. 4. Each of the six curves is based on data from more than $20 \mathrm{GA}$ runs. Clearly the binary approach is unable to effectively search the size $2^{256}$ search space. Meanwhile, even the evolution of the worst quadtree is able to significantly outperform the best binary evolution. Part of the effect of the hierarchical, variable-length quadtree genome is seen in Figs. 5, 6. The highest fitness individuals after 100 generations typically have a number of terminal nodes below 25 , and an average metal patch size that is about 16 times greater than 


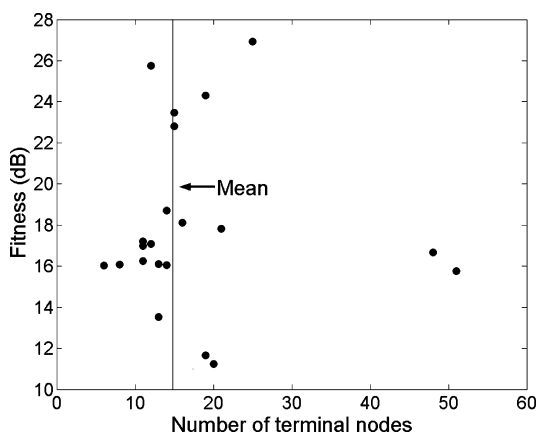

Fig. 5. Number of terminal nodes in quadtree-based designs.

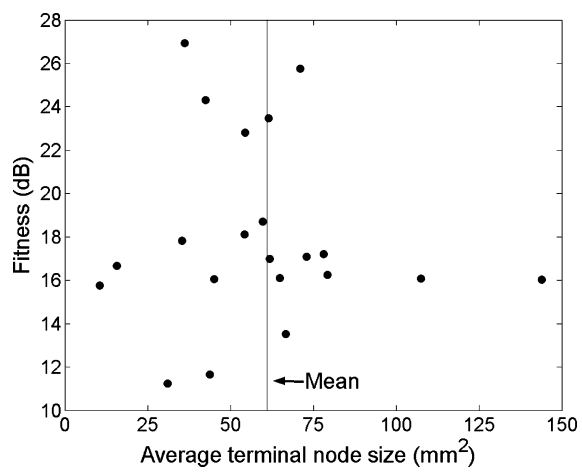

Fig. 6. Average size of terminal node metal in quadtree-based designs.

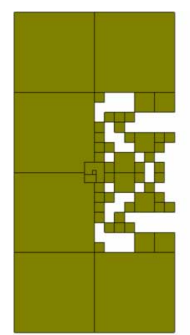

(a)

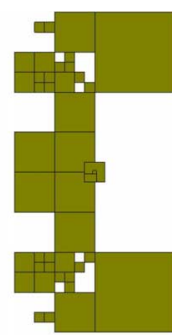

(b) (c)

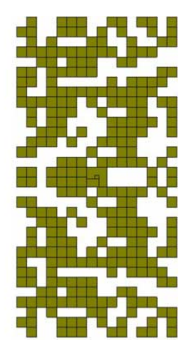

Fig. 7. Comparison of generated patch designs. Best quadtree design (a). Worst quadtree design (b). Best binary design (c).

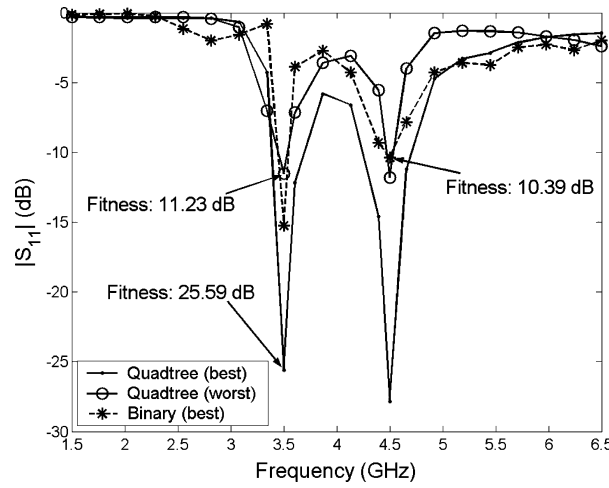

Fig. 8. Antenna performance corresponding to Fig. 7 designs.

the standard $2 \times 2 \mathrm{~mm}$ binary cell. This is in contrast to the fully populated 256 bits that the binary encoding is continually operating on. Fig. 8 shows the performance over a frequency range

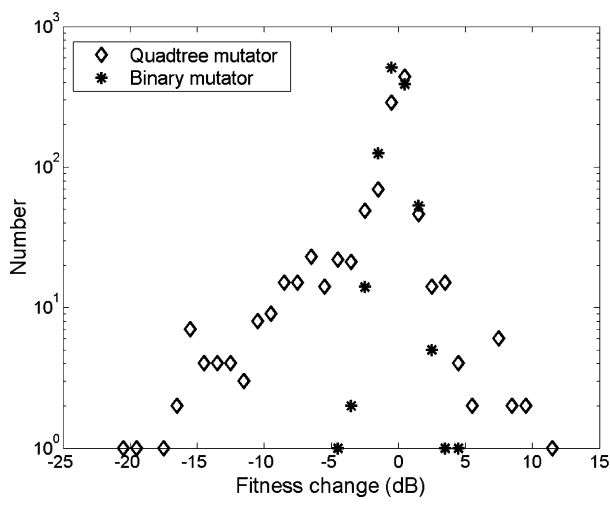

Fig. 9. Comparison of fitness changes caused by mutation operators.

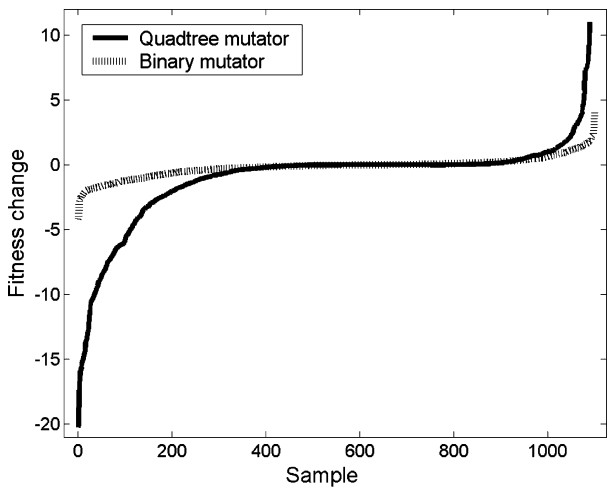

Fig. 10. Comparison of (ordered) fitness changes caused by mutation operators.

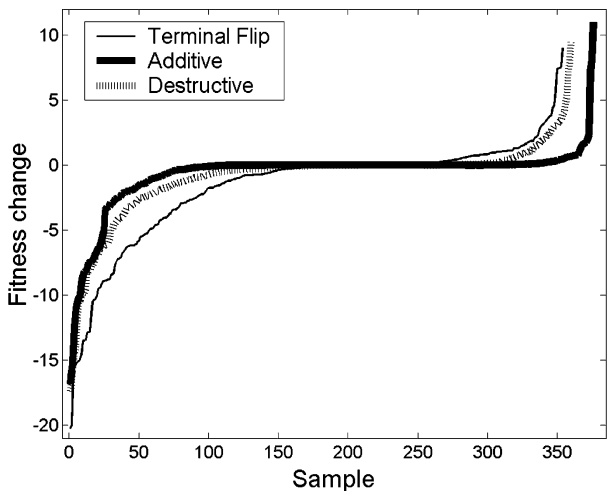

Fig. 11. Comparison of (ordered) fitness changes caused by mutation routines within quadtree mutation operator.

corresponding to the designs given in Fig. 7. Most of the generated binary designs were fragmented and perhaps more difficult to manufacture [e.g., Fig. 7(c)].

Figs. 9, 10 explore the effect of the mutation operator for about 1000 mutations. Fig. 9 is based on the number of "hits" in a fitness change bin. While the standard flip mutator of the binary genome is narrowly distributed around 0 , the quadtree mutator implemented for this study is able to create much greater perturbations to the upside. In Fig. 10, the data is first ordered in terms of increasing fitness change. We see that there are perhaps 200 samples (downside) and 100 samples (upside) that produce a meaningful change to the design for both mutators. Many of these $\sim 100$ fitness improvements caused by a single quadtree 


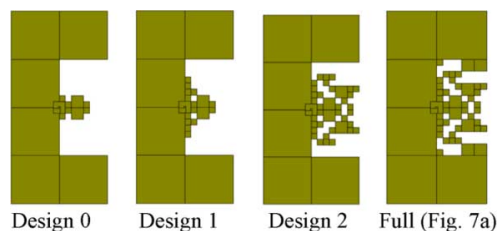

Fig. 12. Intermediate steps leading to full quadtree design.

TABLE II

PERFormanCE OF INTERMEDIATE AND Full DESIGNS

\begin{tabular}{ccc}
\hline Design & $\begin{array}{c}\left|\mathrm{S}_{11}\right|(\mathrm{dB}) \\
(3.5 \mathrm{GHz})\end{array}$ & $\begin{array}{c}\left|\mathrm{S}_{11}\right|(\mathrm{dB}) \\
(4.5 \mathrm{GHz})\end{array}$ \\
\hline Design 0 & -7.43 & -17.27 \\
\hline Design 1 & -8.15 & -16.84 \\
\hline Design 2 & -8.23 & -26.76 \\
\hline Full (Fig. 7a) & -25.59 & -27.85 \\
\hline
\end{tabular}

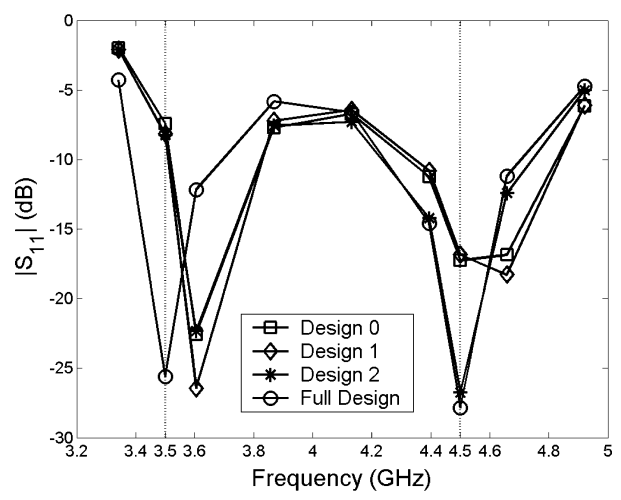

Fig. 13. Performance of antenna designs shown in Fig. 12.

mutation (fraction of a second) are greater than the expected fitness values from a standard binary GA after $95.2 \mathrm{~min}$ of computation (6.52 dB in Table I).

The behavior of the component routines within the quadtree mutator is provided in Fig. 11. The "terminal flip" mutator is directly analogous to the standard binary flip mutator except that it operates on terminal nodes of the variable-size tree. It does not suffer from the limited success rate of the standard binary mutator, suggesting that the novel genome structure and mutator work well in tandem.

\section{ENHANCEMENTS To E-ShaPed PATCh ANTENNA}

It is interesting to note that the most successful designs that were evolved [e.g., Fig. 7(a)] are actually a rediscovery of the well-known E-shaped antenna with enhancements. In this section we investigate how significant are the enhancements to the overall performance. This is achieved by starting with a design resembling the E-shaped patch, and incrementally adding features until the design of Fig. 7(a) is obtained. Fig. 12 shows the sequence of increments. As seen in Fig. 13, "Design 0" and "Design 1" have comparable performance, but are relatively poor at the lower $3.5 \mathrm{GHz}$ design frequency. The resonances appear to be shifted by $\sim 0.1 \mathrm{GHz}$ from the desired locations. "Design 2" improves on the depth and location of the higher resonance, but the $3.5 \mathrm{GHz}$ performance continues to be disappointing. The "Full Design" achieves excellent depth and location of resonances for both 3.5 and $4.5 \mathrm{GHz}$ (Table II, Fig. 13). By comparing "Design 2" with the "Full Design" in Fig. 12, it seems that the improvement in depth and location at $3.5 \mathrm{GHz}$ comes from the inclusion of 6 cells ( 3 upper and 3 lower), which are not present in the standard E-shaped patch [3]. While the series of steps in Fig. 12 is surely not the one that was followed by the GA, it does suggest that the enhancements to the usual E-shaped patch are important to the success of the final design.

There are of course limits to manufacturing. Cells that are connected only at isolated points to the rest of the patch may be problematic for several reasons.

For example, imprecise etching may lead to small channels which allow current into the vertex-connected regions and lead to discrepancies with the current distribution from method-ofmoments simulation software [5]. Thus, it is advisable to verify the performance of final designs with prototypes. Alternatively, the fitness function may be modified so that problematic features are avoided. It appears that a side-effect of the quadtree-based genome is a reduction in fragmentation, which is welcome from the standpoint of manufacturing.

\section{CONCLUSION}

This work has attempted to demonstrate that significant performance advantages can be obtained by carefully designing the genome and genetic operators within GAs. A novel genome structure and genetic operators were presented and shown to routinely outperform the standard mapping by almost a factor of three. As well, they were able to rediscover and improve upon a well-known configuration. The final designs were less fragmented than those from the standard approach, and thus seem easier to manufacture. The variable-length quadtree-based genome has the potential to become increasingly valuable as more general, non-parametric design strategies appear and the search spaces become larger.

\section{ACKNOWLEDGMENT}

This work was supported in part by the Natural Sciences and Engineering Research Council of Canada and Le Fonds québécois de la recherche sur la nature et les technologies.

\section{REFERENCES}

[1] A. Gallardo and D. A. Lowther, "Some aspects of niching genetic algorithms applied to electromagnetic device optimization," IEEE Trans. Magn, vol. 36, no. 4, pp. 1076-1079, 2000.

[2] K. O. Stanley and R. Miikkulainen, "A taxonomy for artificial embryogeny," Artificial Life, vol. 9, no. 2, pp. 93-130, 2003.

[3] F. Y. Yang, X.-X. Zhang, X. Ye, and Y. Rahmat-Samii, "Wide-band E-shaped patch antennas for wireless communications," IEEE Trans. Antennas Propagat., vol. 49, no. 7, pp. 1094-1100, 2001.

[4] M. Dorica and D. D. Giannacopoulos, "Response surface space mapping for electromagnetic optimization," IEEE Trans. Magn, vol. 42, no. 4, pp. 1123-1126, 2006.

[5] F. J. Villegas, T. Cwik, Y. Rahmat-Samii, and M. Manteghi, "A parallel electromagnetic genetic-algorithm optimization (EGO) application for patch antenna design," IEEE Trans. Antennas Propagat., vol. 52, no. 9 , pp. 2424-2435, 2004.

[6] J. M. Johnson and Y. Rahmat-Samii, "Genetic algorithms and method of moments (GA/MOM) for the design of integrated antennas," IEEE Trans. Antennas Propagat., vol. 47, no. 10, pp. 1606-1614, 1999.

[7] W. Wang, Y. Lu, J. S. Fu, and Y. Z. Xiong, "Particle swarm optimization and finite element based approach for microwave filter design," IEEE Trans. Magn., vol. 41, no. 5, pp. 1800-1803, 2005.
Manuscript received April 24, 2006 (e-mail: dennis.giannacopoulos@mcgill. ). 